OPEN ACCESS

Edited by:

Hiroshi Iwata,

Juntendo University, Japan

Reviewed by:

Giuseppe Militello,

Mirimus Inc., United States

Mabruka Alfaidi,

Louisiana State University Health

Shreveport, United States

*Correspondence:

Gang L

ligang20210203@163.com

Specialty section:

This article was submitted to

Atherosclerosis and Vascular

Medicine,

a section of the journal

Frontiers in Cardiovascular Medicine

Received: 19 March 2021

Accepted: 25 May 2021

Published: 21 June 2021

Citation:

Guo F, Sha Y, Hu B and Li G (2021) Correlation of Long Non-coding RNA LnCRNA-FA2H-2 With Inflammatory

Markers in the Peripheral Blood of Patients With Coronary Heart Disease.

Front. Cardiovasc. Med. 8:682959.

doi: 10.3389/fcvm.2021.682959

\section{Correlation of Long Non-coding RNA LncRNA-FA2H-2 With Inflammatory Markers in the Peripheral Blood of Patients With Coronary Heart Disease}

\author{
Fengxia Guo ${ }^{1}$, Yanhua Sha ${ }^{2}$, Bing $\mathrm{Hu}^{3}$ and Gang $\mathrm{Li}^{1 *}$ \\ ${ }^{1}$ Department of Clinical Laboratory, Henan Provincial People's Hospital, People's Hospital of Zhengzhou University, \\ Zhengzhou, China, ${ }^{2}$ Department of Laboratory Medicine, The Second Affiliated Hospital of Guangzhou University of Chinese \\ Medicine, Guangzhou, China, ${ }^{3}$ Department of Clinical Laboratory, Affiliated Cancer Hospital of Zhengzhou University, \\ Zhengzhou, China
}

Objective: To characterize the expression of long non-coding RNA LncRNA-FA2H-2 in coronary heart disease $(\mathrm{CHD})$ and its correlation with inflammatory markers.

Methods: From December 2018 to December 2020, 316 patients at Henan Provincial People's Hospital who complained of chest tightness or chest pain and had coronary angiography to clarify their coronary artery conditions for definitive diagnoses were selected as the study subjects. Plasma was collected to detect white blood cells (WBCs), total cholesterol (TG), triglyceride cholesterol (TC), low-density lipoprotein cholesterol (LDL-C), high-density lipoprotein cholesterol (HDL-C), apolipoprotein A1 (ApoA1), and C-reactive protein (CRP) levels. Tumor necrosis factor (TNF- $\alpha$ ), monocyte chemotactic protein 1 (MCP-1), vascular cell adhesion molecule-1 (VCAM-1), intercellular cell adhesion molecule-1 (ICAM-1), and interleukin-6 (IL-6) levels were also measured using ELISA. The expression levels of IncRNA-FA2H-2 were measured using quantitative real-time PCR. The data obtained were analyzed by independent sample $t$-tests, rank sum tests, regression analyses, Pearson's or Spearman's correlation analyses, and receiver operating characteristic curves.

Results: (1) Compared with the control group, the differences in age, sex, diabetes, smoking, drinking, body mass index (BMI), WBC, TC, and LDL-C in CHD were not statistically significant, while the differences in hypertension, TG, HDL-C, ApoA1, and CRP were statistically significant. (2) In the grouping of coronary lesion branches, patients with age, sex, hypertension, diabetes, smoking, drinking, BMI, WBC, TC, LDL-C, HDL-C, and ApoA1 differences were not statistically significant, but TG and CRP differences were statistically significant. (3) The relative expressions of TNF- $\alpha$, MCP-1, VCAM-1, ICAM-1, and IL-6 were significantly upregulated in the CHD group $(P<0.001)$. (4) The results showed that the relative levels of TNF- $\alpha$, MCP-1, VCAM-1, ICAM-1, and IL-6 between the two comparative analyses (high risk, moderate risk, and low risk groups) were statistically significant. In addition, positive correlations were found between the Gensini score and TNF- $\alpha$, MCP-1, VCAM-1, ICAM-1, and IL-6 in CHD patients. 
(5) LncRNA-FA2H-2 relative expression in the $\mathrm{CHD}$ group was significantly downregulated $(P<0.001)$. (6) The differences in the expression levels of LncRNA-FA2H-2 were statistically significant between the two comparative analyses $(P<0.01)$, except between the 2-branch lesion and 3-branch lesion groups. (7) LncRNA-FA2H-2 was not associated with age, sex, hypertension, diabetes, smoking, drinking, BMI, WBC, TG, TC, LDL-C, HDL-C, and ApoA1 ( $>$ > 0.05). (8) A correlation was found between LnCRNA-FA2H-2 and MCP-1, and VCAM-1, ICAM-1, IL-6, and Gensini. (9) The results indicated that the relative levels of LncRNA-FA2H-2 between the two comparative analyses (high risk, moderate risk, and low risk groups) were statistically significant. A negative correlation was found between the Gensini score and LncRNA-FA2H-2. (10) ROC curve analyses of TNF- $\alpha$, MCP-1, VCAM-1, ICAM-1, and IL-6 in CHD showed the area under the curve $(A \cup C)=0.832(0.77,0.893)$ with a cut-off value of 290.5, a sensitivity of $73 \%$, and a specificity of $64 \%$; AUC $=0.731$ $(0.653,0.809)$ with a cut-off value of 396 and with a sensitivity of $59 \%$ and specificity of $79 \%$; AUC $=0.822(0.757,0.887)$ with a cut-off value of 264 and with a sensitivity of $72 \%$ and specificity of $83 \%$; AUC $=0.794(0.715,0.874)$ with a cut-off value of 201.5 and with a sensitivity of $75 \%$ and specificity of $65 \%$; $A \cup C=0.760(0.685,0.834)$ with a cut-off value of 328 and with a sensitivity of $55 \%$ and specificity of $90 \%$. (11) ROC curve analysis of LncRNA-FA2H-2 in CHD patients showed AUC $=0.834(0.688,0.85)$ with a cut-off value of 3.155 and with a sensitivity of $85 \%$ and specificity of $82 \%$. (12) Logistic analyses showed that TNF- $\alpha$, MCP-1, VCAM-1, IL-6, and LnCRNA-FA2H-2 were independent risk factors for $\mathrm{CHD}$.

Conclusions: The expression of LncRNA-FA2H-2 was reduced and inversely correlated with inflammation-related factors in CHD patients. LncRNA-FA2H-2 may have potential as an inflammatory marker for risk assessment of $\mathrm{CHD}$ development.

Keywords: coronary heart disease, long non-coding RNA, inflammatory marker, LncRNA-FA2H-2, peripheral blood

\section{INTRODUCTION}

It is well-known that coronary heart disease (CHD) is a prevalent cardiovascular disorder $(1,2)$ and is the main cause of cardiovascular diseases, while the inflammatory response is present throughout the course of CHD (3-5). Coronary angiography is currently the main method of diagnosing CHD, but the tests are expensive and invasive, and unsuitable for dynamic monitoring. However, blood biochemical tests provide an important laboratory basis for the diagnosis and treatment of heart disease, especially CHD. Previous studies have shown that the levels of inflammatory biomarkers can assess the occurrence and severity of CHD (6-8), but these inflammatory indicators are susceptible to interference by the body's environment and external factors, and are therefore, unstable as indicators. An in-depth investigation of potential biomarkers of CHD could therefore be an important guide to assessing the risk of CHD.

Most (90\%) of the transcribable genes in the human genome are non-coding RNAs (ncRNAs), and only $2 \%$ are protein-coding genes (9). Current research on specific long non-coding RNAs (lncRNAs) has mainly focused on tumor diseases, but the role of lncRNAs in cardiovascular diseases and its related mechanisms are not yet known. As functional studies of lncRNAs have increased, investigators have found that some lncRNAs can be used as potential target genes and biomarkers for the prevention and treatment of CHD. The current studies of LncRNAs related to CHD mainly focus on RNCR3, UCA1, LncRNAp21, HOTAIR, ANRIL, and TUG1 (10-16). The lncRNA, RNAANRIL (CDKN2B-AS), localized on human chromosome 3, was found to be strongly correlated with the risk of CHD (17). In addition, LncRNA CARMEN and LncRNA fendrr are associated with embryonic heart development $(18,19)$. LncRNAAng362 may be associated with prognosis after coronary stenting (percutaneous coronary intervention) for CHD (20). Increased expression of LncRNA GAS5 was found in plaques of atherosclerotic individuals, and the mechanism may be related to exosome regulation of macrophage and endothelial cell apoptosis in atherosclerotic plaques (21). LncRNA MALAT1 was positively correlated with the expression of inflammatory mediators, IL-6 and TNF, and the mechanism may be related to the inflammatory ligand, serum amyloid 3, in endothelial cells (22). Research on LncRNAs and CHD is in the developing stage, and further studies are needed before it can be clinically used. LncRNA-FA2H2 is a newly identified inflammation-related LncRNA reported 
in our previous study, and it attenuated the development of atherosclerosis via inhibition of the inflammatory response (23), but its expression in CHD patients is still unclear. In the present study, we characterized the expression of LncRNA-FA2H-2 in CHD patients, as well as its correlation with inflammatory markers, and we determined whether LncRNA-FA2H-2 could be used as a biomarker for predicting the risk of $\mathrm{CHD}$.

\section{RESEARCH SUBJECTS AND METHODS}

\section{Study Subjects}

This study examined patients admitted to our hospital with chest tightness or chest pain as the main complaint for definitive diagnosis between December 2018 and December 2020. The patients underwent a series of clinical examinations, and coronary angiography was finally conducted to clarify the condition of their coronary arteries. These patients were selected for the study subjects, and were divided into the control and $\mathrm{CHD}$ groups. The inclusion criteria for the $\mathrm{CHD}$ group included patients with at least one artery (left main coronary artery trunk, left anterior descending branch, left circumflex branch, and right coronary artery) having more than 50\% stenosis. Inclusion criteria for the control group included coronary angiography showing no stenosis or only myocardial bridge changes (congenital anomalies of coronary artery development, which refers to a segment of the coronary artery that travels through the myocardium and is covered by a bundle of myocardial fibers called a myocardial bridge) in the coronary artery. Exclusion criteria included previous coronary surgery, including angiography and bypass surgery, active bleeding from various causes, with severe liver and kidney insufficiency, peripheral vascular disease, tumors, hematological diseases, acute and chronic infectious diseases, chronic obstructive pulmonary disease, cardiac arrhythmias, and diabetes mellitus. The study was approved by the hospital ethics committee, and each patient signed an informed consent form.

\section{Specimen Collection and Processing}

Blood samples were taken before the administration of any drugs to the patients. We drew $7 \mathrm{ml}$ of fasting venous blood from the patients in the morning and placed the samples in EDTA tubes in a temperature-controlled centrifuge. The samples were centrifuged at 3,000 rpm for $15 \mathrm{~min}$. The plasma was retained for subsequent studies, and the remaining samples were stored at $-80^{\circ} \mathrm{C}$ for cryopreservation. The expressions of $\mathrm{WBC}, \mathrm{TG}$, TC, LDL-C, HDL-C, ApoA1, and CRP in the plasma were subsequently measured.

\section{METHODS}

\section{ELISA}

The plasma from patients was collected, and the levels of TNF- $\alpha$ (ab183218), MCP-1 (ab179886), VCAM-1 (ab134047), ICAM-1 (ab174445), and IL-6 (ab178013) were measured by ELISA kits purchased from Abcam (Cambridge, MA, USA). All experimental procedures were performed in accordance with the manufacturer's instructions. The absorbance values were measured using a microplate reader (Molecular Devices, San Jose, CA, USA) at a wavelength of $450 \mathrm{~nm}$.

\section{The qRT-PCT}

Total RNA was isolated from plasma using a TRIzol kit (Invitrogen, Carlsbad, CA, USA). The RNA concentration was measured with a spectrophotometer (NanoDrop ${ }^{\circledR}$ 2000; Thermo Fisher Scientific, Waltham, MA, USA). First strand complementary DNA (cDNA) of lncRNA-FA2H-2 was synthesized using M-MLV reverse transcriptase (Promega, Madison, WI, USA), while total mRNA was synthesized into cDNA using a High Capacity cDNA Reverse Transcription Kit (Thermo Fisher Scientific), both in a reaction volume of 20 $\mu \mathrm{l}$ containing $1 \mu \mathrm{g}$ of total RNA as the template strand. The expression levels of lncRNA-FA2H-2 and U6 as endogenous controls were evaluated by RT-qPCR using the SYBR Green Master Mix (TaKaRa Bio, Beijing, China) on a LightCycler 480 Fast Real-Time PCR system (Roche Molecular Systems, Pleasanton, CA, USA). PCR reactions were run in a $10 \mu \mathrm{l}$ final volume containing $100 \mathrm{ng}$ CDNA, $0.8 \mu \mathrm{l}$ forward and reverse primers, $5 \mu \mathrm{l}$ SYBR-Green, and $3.2 \mu \mathrm{l}$ ddH2O. Ploidy differences in expression levels were determined using the $2^{-\Delta \Delta \mathrm{Ct}}$ method. All experimental procedures were performed according to the manufacturer's instructions. All samples were measured in triplicate and the mean values were used for comparative analysis. All primers used for RT-PCR were synthesized by Sangon Biotech (Shanghai, China). The RT-qPCR thermal cycling procedure consisted of an initial pre-incubation step of $20 \mathrm{~s}$ at $95^{\circ} \mathrm{C}$ followed by 40 cycles of $10 \mathrm{~s}$ at $95^{\circ} \mathrm{C}$, $20 \mathrm{~s}$ at $60^{\circ} \mathrm{C}$, and $1 \mathrm{~s}$ at $70^{\circ} \mathrm{C}$. The U6 primer sequences were as follows: forward, GTGGCCGAGGACTTTGATTG, reverse, CCTGTAACAACGCATCTCATATT; LncRNAFA2H-2: forward, TTCCCTTACTCAGTGGTTCCC, reverse, GCTTTCTCCAATCCTACC.

\section{Gensini Score}

Two or more experienced investigators quantitatively scored the degree of stenosis in the four vessels of the left main coronary artery trunk, left circumflex branch, right coronary artery, and left anterior descending branch based on the imaging results using the current internationally accepted method of visual diameter measurements. The score representing the degree of coronary lesion in each patient was the sum of the scores of each branch.

\begin{tabular}{lclc}
\hline Narrowness & Score & Lesion & Score \\
\hline $1-25 \%$ & 1 & $\begin{array}{l}\text { Left main stem } \\
\text { Left anterior descending branch or left }\end{array}$ & 5 \\
26-50\% & 2 & $\begin{array}{l}\text { gyral branch } \\
\text { Middle left anterior descending branch }\end{array}$ & 1.5 \\
$51-75 \%$ & 4 & $\begin{array}{l}\text { Distal segment of the left anterior } \\
\text { descending branch }\end{array}$ & 1.0 \\
$76-90 \%$ & 8 & $\begin{array}{l}\text { Middle and distal left gyral branch } \\
\text { Right coronary artery }\end{array}$ & 1.0 \\
F1-99\% & 16 & 1 \\
Fully closed & 32 & Small branch & 0.5
\end{tabular}


TABLE 1 | Comparison of clinical data.

\begin{tabular}{lccc}
\hline & Control $(\boldsymbol{n}=\mathbf{1 0 0})$ & CHD $(\boldsymbol{n}=\mathbf{2 1 6})$ & $\boldsymbol{P}$ \\
\hline Age (year) & $55.8 \pm 4.4$ & $56.1 \pm 4.1$ & 0.669 \\
Sex & $28 / 22$ & $57 / 49$ & 0.54 \\
Hypertension [case (\%)] & $29(29 \%)$ & $105(48.6 \%)$ & 0.001 \\
Diabetes [case (\%)] & $35(35 \%)$ & $89(41.2 \%)$ & 0.089 \\
Smoking [case (\%)] & $34(34 \%)$ & $94(43.5 \%)$ & 0.102 \\
Drinking [case (\%)] & $40(40 \%)$ & $99(45.8 \%)$ & 0.092 \\
BMl (Kg/m $\left.{ }^{2}\right)$ & $22.18 \pm 2.32$ & $23.21 \pm 2.45$ & 0.165 \\
WBC (10 $)$ & $5.23 \pm 1.83$ & $5.39 \pm 1.75$ & 0.318 \\
TG $(\mathrm{mmol} / \mathrm{L})$ & $3.59 \pm 0.83$ & $4.90 \pm 0.82$ & 0.001 \\
TC $(\mathrm{mmol} / \mathrm{L})$ & $0.85 \pm 0.38$ & $0.84 \pm 0.38$ & 0.9 \\
LDL-C (mmol/L) & $2.42 \pm 0.43$ & $2.38 \pm 0.47$ & 0.619 \\
HDL-C (mmol/L) & $1.71 \pm 0.71$ & $1.07 \pm 0.2$ & 0.001 \\
ApoA1 (g/L) & $1.32 \pm 0.29$ & $1.0 \pm 0.25$ & 0.001 \\
CRP (mg/dL) & $4.07 \pm 2.32$ & $21.66 \pm 5.86$ & 0.001 \\
\hline
\end{tabular}

\section{STATISTICAL METHODS}

Statistical analysis was performed using SPSS statistical software for Windows, version 23.0 (SPSS, Chicago, IL, USA). The Kolmogorov-Smirnov test was used for normal distributions, and conformity to a normal distribution was expressed as the mean \pm standard deviation. The independent samples $t$-test was used for comparisons between two groups for measurement data, and one-way ANOVA was used for comparisons between multiple groups. Failure to conform to a normal distribution was expressed as the median percentile.

Comparisons between two groups were performed using the Mann-Whitney $U$ rank sum test, and multiple comparisons were conducted using the Kruskal-Wallis rank sum test. Pearson's or Spearman's correlation analyses, ROC curves, and logistic regression analyses of risk factors for $\mathrm{CHD}$ patients were also used. A value of $P<0.05$ indicated a statistically significant difference.

\section{RESULTS}

\section{Analysis of Clinical Data}

The differences in age, sex, diabetes, smoking, drinking, BMI, WBC, TC, and LDL-C in the CHD group were not statistically significant $(P>0.05)$, but the differences in hypertension, TG, HDL-C, ApoA1, and CRP levels were statistically significant $(P<0.001)$ (Table 1).

\section{Analysis of Clinical Data in the Grouping of the Number of Coronary Lesions}

$\mathrm{CHD}$ patients were grouped according to the number of coronary lesion branches. There were 72 patients in the 1-branch lesion group, 80 patients in the 2-branch lesion group, and 64 patients in the 3-branch lesion group. The differences in age, sex, hypertension, diabetes, smoking, drinking, BMI, WBC, TC, LDL$\mathrm{C}, \mathrm{HDL}-\mathrm{C}$, and ApoA1 were not statistically significant in the three groups, but the differences in TG and CRP were statistically significant $(P<0.001)$ (Table 2$)$.

\section{Analyses of Expression Levels of TNF- $\alpha$, MCP-1, VCAM-1, ICAM-1, and IL-6 in CHD Patients}

We analyzed the expression levels of TNF- $\alpha$, MCP-1, VCAM1, ICAM-1, and IL-6 using ELISA kits. The expression levels of TNF- $\alpha$, MCP-1, VCAM-1, ICAM-1, and IL- 6 were significantly higher in CHD patients (all, $P<0.001$ ) (Table 3 ).

\section{Correlation Analysis Between the Gensini Score and TNF- $\alpha$, MCP-1, VCAM-1, ICAM-1, and IL-6 in CHD Patients}

CHD patients were grouped into high risk, moderate risk, and low risk groups according to their Gensini score. The results showed that the relative levels of TNF- $\alpha$, MCP-1, VCAM1 , ICAM-1, and IL- 6 between the two comparative analyses were statistically significant (Figure 1). In addition, positive correlations were found between the Gensini score and TNF- $\alpha$, MCP-1, VCAM-1, ICAM-1, and IL-6 in CHD patients (Table 4).

\section{Analysis of the Expression Level of LncRNA-FA2H-2 in CHD Patients}

The relative expression of LncRNA-FA2H-2 was significantly downregulated in $\mathrm{CHD}$ patients when compared with the control group $(P<0.001)$ (Table 5 and Figure 2 ).

\section{Detection of the Expression Level of LncRNA-FA2H-2 in Lesion Branch \\ Groupings}

CHD patients were grouped according to the number of coronary lesion branches, with 72 patients in the 1-branch lesion group, 80 patients in the 2-branch lesion group, and 64 patients in the 3-branch lesion group, while the control group was defined as the 0-branch lesion group. As shown in Table 6 below, the differences between the two comparative analyses were statistically significant $(P<0.01)$, except for no statistical difference between the 2-branch lesion and 3-branch lesion groups.

\section{Correlation Analysis of LncRNA-FA2H-2 With Clinical Characteristics in CHD \\ Patients}

LncRNA-FA2H-2 showed no correlation $(P>0.05)$ with age, sex, hypertension, diabetes, smoking, drinking, BMI, WBC, TG, TC, LDL-C, HDL-C, or ApoA1 (Table 7).

\section{Correlation Analysis Between LncRNA-FA2H-2 and TNF- $\alpha$, MCP-1,} VCAM-1, ICAM-1, and IL-6 in CHD Patients A negative correlation was found between LncRNA-FA2H-2 and MCP-1, VCAM-1, ICAM-1, IL-6 in CHD patients (Table 8). 
TABLE 2 | Analysis of clinical data in the grouping of the number of branches of coronary lesions.

\begin{tabular}{|c|c|c|c|c|}
\hline & 1 Sticks $(n=72)$ & 2 Sticks $(n=80)$ & 3 Sticks $(n=64)$ & $P$ \\
\hline Age (year) & $52.1 \pm 3.8$ & $54.8 \pm 4.2$ & $53.5 \pm 4.4$ & 0.589 \\
\hline Sex (male/female) & $37 / 35$ & $42 / 38$ & $31 / 33$ & 0.47 \\
\hline Hypertension [case (\%)] & 32 (44.4\%) & 38 (47.5\%) & 35 (54.6\%) & 0.352 \\
\hline Diabetes [case (\%)] & $25(34.7 \%)$ & $34(42.5 \%)$ & $30(46.8 \%)$ & 0.673 \\
\hline Smoking [case (\%)] & $30(41.6 \%)$ & 35 (43.7\%) & $29(45.3 \%)$ & 0.701 \\
\hline Drinking [case (\%)] & $38(52.7 \%)$ & $33(41.2 \%)$ & $28(43.7 \%)$ & 0.328 \\
\hline $\mathrm{BMl}\left(\mathrm{Kg} / \mathrm{m}^{2}\right)$ & $23.34 \pm 2.78$ & $23.09 \pm 2.18$ & $22.10 \pm 2.31$ & 0.173 \\
\hline WBC $\left(10^{9}\right)$ & $4.9 \pm 1.67$ & $5.2 \pm 1.85$ & $5.01 \pm 1.54$ & 0.207 \\
\hline TG (mmol/L) & $3.96 \pm 0.45$ & $4.34 \pm 1.00$ & $5.23 \pm 0.83$ & 0.001 \\
\hline TC (mmol/L) & $0.81 \pm 0.42$ & $0.83 \pm 0.57$ & $0.81 \pm 0.33$ & 0.458 \\
\hline LDL-C (mmol/L) & $2.34 \pm 0.23$ & $2.41 \pm 0.32$ & $2.30 \pm 0.46$ & 0.420 \\
\hline HDL-C (mmol/L) & $1.65 \pm 0.39$ & $1.74 \pm 0.43$ & $1.68 \pm 0.54$ & 0.314 \\
\hline ApoA1 (g/L) & $1.29 \pm 0.17$ & $1.21 \pm 0.28$ & $1.26 \pm 0.32$ & 0.183 \\
\hline $\mathrm{CRP}(\mathrm{mg} / \mathrm{dL})$ & $10.23 \pm 1.30$ & $19.34 \pm 3.29$ & $23.29 \pm 1.7$ & 0.001 \\
\hline
\end{tabular}

TABLE 3 | Comparison of inflammation-related factors.

\begin{tabular}{lccc}
\hline & Control $(\boldsymbol{n}=\mathbf{1 0 0})$ P50 (P25, P75) & CHD $(\boldsymbol{n}=\mathbf{2 1 6 )}$ P50 (P25, P75) & $\boldsymbol{P}$ \\
\hline TNF- $\alpha(\mathrm{pg} / \mathrm{ml})$ & $213(95.75,276)$ & $393(257.75,432)$ & $<0.001$ \\
MCP-1 $(\mathrm{pg} / \mathrm{ml})$ & $327(120.25,360)$ & $418.5(271.75,538.75)$ & $<0.001$ \\
VCAM-1 $(\mathrm{pg} / \mathrm{ml})$ & $172.5(108,192)$ & $320(230,384.75)$ & $<0.001$ \\
ICAM-1 $(\mathrm{pg} / \mathrm{ml})$ & $138(109,183)$ & $294(196.5,322.5)$ & $<0.001$ \\
IL-6 $(\mathrm{pg} / \mathrm{ml})$ & $193.5(110.5,285.25)$ & $354(202.25,467)$ & $<0.001$ \\
\hline
\end{tabular}

\section{Correlation Analysis Between the Gensini Score and LncRNA-FA2H-2 in CHD \\ Patients}

CHD patients were grouped into high risk, moderate risk, and low risk groups according to their Gensini score. The data showed that the relative levels of LncRNA-FA2H-2 between the two comparative analyses were statistically significant (Figure 3). A negative correlation was found between the Gensini score and LncRNA-FA2H-2 (Table 9).

\section{Comparison of Sensitivities and Specificities of ROC Curves for TNF- $\alpha$, MCP-1, VCAM-1, ICAM-1, and IL-6}

By performing ROC curves for CHD patients, the cut-off value, sensitivity, and specificity corresponding to the maximum of the Jorden index were calculated. The results revealed that the cut-off value of TNF- $\alpha$ was 290.5 , with a sensitivity of $73 \%$ and specificity of $64 \%$, and the AUC was 0.832 (0.77, 0.893); the cut-off value of MCP-1 was 396, with a sensitivity of $59 \%$ and specificity of $79 \%$, and the AUC was $0.731(0.653,0.809)$; the cut-off value of VCAM- 1 was 264 , with a sensitivity of $72 \%$ and specificity of $83 \%$, the AUC was $0.822(0.757,0.887)$; the cut-off value of ICAM-1 was 201.5, with a sensitivity of $75 \%$ and specificity of $65 \%$, the AUC was $0.794(0.715,0.874)$; the cut-off value of IL- 6 was 328 , with a sensitivity of $55 \%$ and specificity of $90 \%$ and the AUC was $0.760(0.685,0.834)$ (Table 10).

\section{ROC Curve Analyses of LncRNA-FA2H-2 Sensitivity and Specificity}

The ROC curve was performed for CHD patients, and the corresponding critical value, sensitivity, and specificities at the maximum of the Jorden index were calculated. The results indicated that the cut-off value of LncRNA-FA2H-2 was 3.155, with a sensitivity of $85 \%$ and specificity of $82 \%$, and the AUC was $0.834(0.688,0.85)$ (Table 11).

\section{Binary Logistic Regression Analysis}

$\mathrm{CHD}$ was used as the dependent variable, inflammation-related factors were included as independent variables, and the values assigned for binary logistic regression analysis showed that TNF- $\alpha$, MCP-1, VCAM-1, IL-6, and LncRNA-FA2H-2 were independent risk factors for CHD patients (Table 12).

\section{DISCUSSION}

The study of pathophysiological changes in CHD patients has shown that inflammatory responses play a key role in the course of CHD. A variety of inflammatory markers are not only present in the walls of sclerotic arteries, but also detectable in the blood, and these inflammatory markers can be used not only for predicting the risk of developing CHD, but also for the evaluation of disease outcomes (24). The selection of inflammatory markers is therefore important for the risk assessment of CHD. Previous studies have shown 


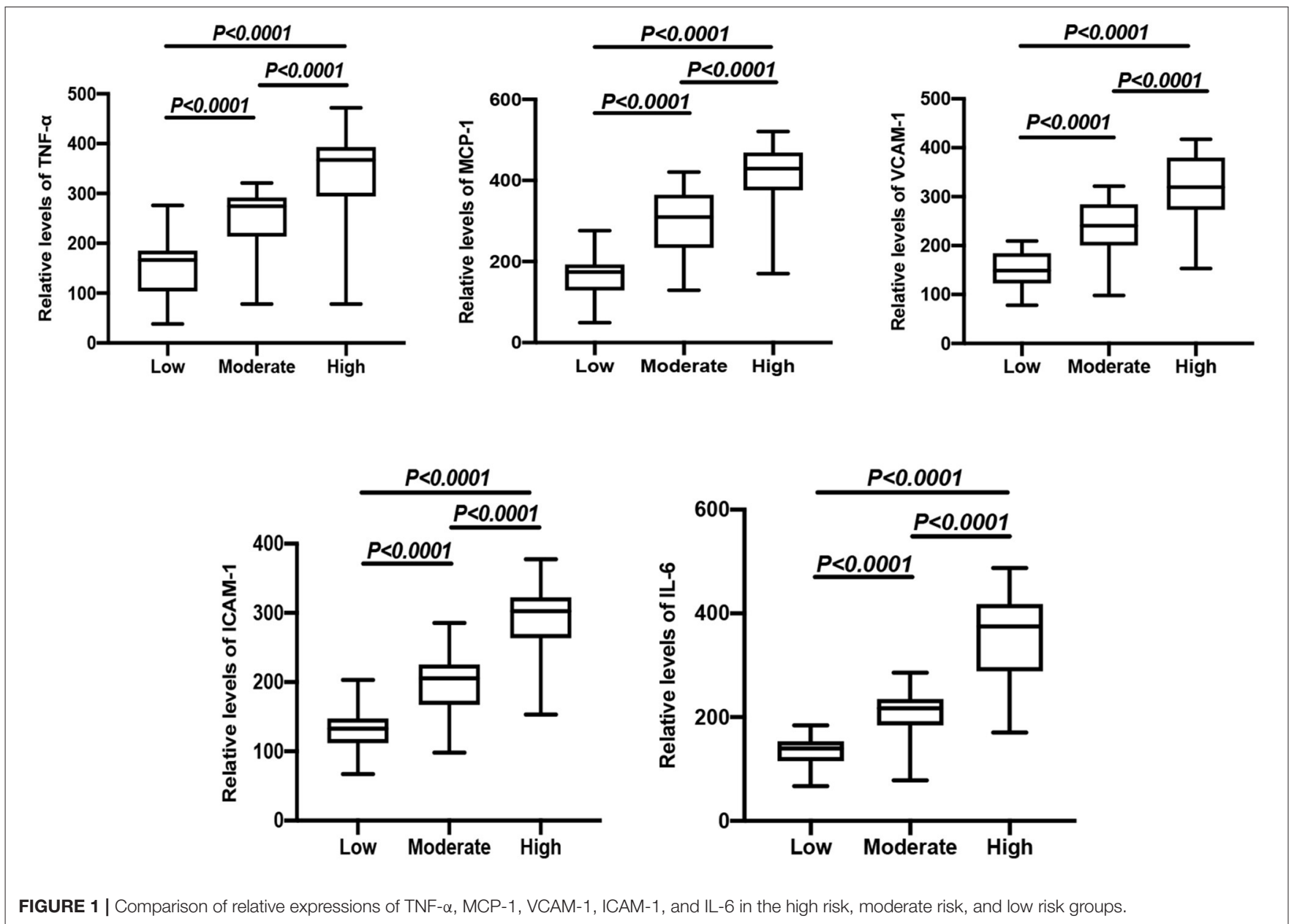

TABLE 4 | Correlation analysis of the Gensini score and TNF- $\alpha$, MCP-1, VCAM-1, ICAM-1, and IL-6.

\begin{tabular}{lcc}
\hline & \multicolumn{2}{c}{ Gensini score } \\
\cline { 2 - 3 } & $\mathbf{R}$ & $\boldsymbol{P}$ \\
\hline TNF- $\alpha$ & 0.231 & 0.045 \\
MCP-1 & 0.218 & 0.032 \\
VCAM-1 & 0.173 & 0.029 \\
ICAM-1 & 0.157 & 0.020 \\
IL-6 & 0.225 & 0.013 \\
\hline
\end{tabular}

that some inflammatory markers have important prognostic values for CHD, such as hs-CRP, NLR, IL-8, and IL-10 (25, 26). However, these inflammatory indicators are susceptible to external environmental interference and cannot accurately assess the magnitude of risk for CHD patients. Therefore, the search for reliable and novel inflammatory markers can help to detect cardiovascular disease early and facilitate timely disease monitoring and prognostic assessment.

LncRNAs, which are mainly localized in the cytoplasm or nucleus and do not have a protein-coding function in addition to lacking an obvious open reading frame, have been shown to be key factors in biological processes (21). LncRNAs have been found to be present in whole blood, serum and plasma, breast milk, and urine, and lncRNA was found to remain stable in harsh conditions, such as boiling, extreme $\mathrm{pH}$, and low temperature (27). The stable nature of lncRNAs makes it possible to use them clinically to monitor the pathophysiological status of patients. There is growing evidence that lncRNAs can be used as important biomarkers for risk assessment in cardiovascular disease and may be better indicators among the non-invasive indicators to assess the magnitude of CHD risk. For example, LncRNA ANRIL, and lncRNA H19 are strongly associated with the risk of developing coronary atherosclerosis (28). In addition, RP5-833A20.1 plays a role in promoting atherosclerosis by increasing the expressions of LDL-C and inflammatory factors, and decreasing the expression of HDL-C (29). Studies have confirmed that lncRNA LIPCAR is closely associated with the prognoses of heart failure patients (30). The lncRNA Meg3 is expected to be a new target for the treatment of cardiac remodeling and myocardial fibrosis (31). The lncRNA coronary marker is significantly elevated in the plasma of patients with CHD (32). Another study reported that lncRNA aHIF, ANRIL, and Kcnq1ot1 were closely related with the occurrence of acute myocardial infarction, and that 
TABLE 5 | Analysis the relative expression of LncRNA-FA2H-2.

\begin{tabular}{|c|c|c|c|}
\hline & Control $(n=100)$ P50 (P25, P75) & CHD $(n=216)$ P50 (P25, P75) & $P$ \\
\hline LncRNA-FA2H-2 & $3.24(2.17,4.1)$ & $1.57(1.26,2.46)$ & $<0.001$ \\
\hline
\end{tabular}

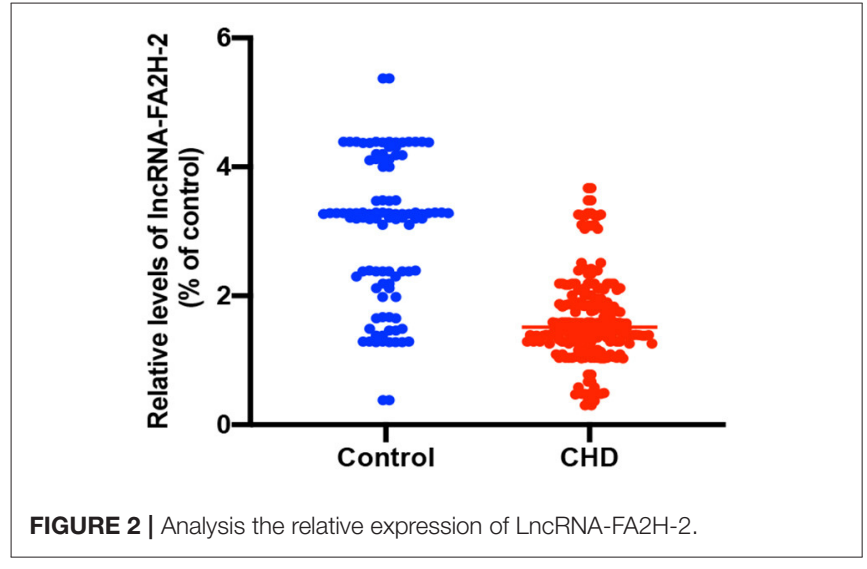

TABLE 6 | The expression levels of LncRNA-FA2H-2 in lesion branch number subgroups.

\begin{tabular}{lc}
\hline Number of sticks & LncRNA-FA2H-2 \\
\hline 0 & $3.24(2.17,4.1)^{\mathrm{bcd}}$ \\
1 & $1.98(1.59,2.13)^{\mathrm{acd}}$ \\
2 & $1.59(1.26,1.89)^{\mathrm{ab}}$ \\
3 & $1.27(1.26,2.34)^{\mathrm{ab}}$
\end{tabular}

For a compared with the 0 -branch lesion group, $P<0.01$ was statistically significant; for $b$ compared with the 1-branch lesion group, $P<0.01$ was statistically significant; for $C$ compared with the 2-branch lesion group, $P<0.01$ was statistically significant; and for $d$ compared with the 3-branch lesion group, $P<0.01$ was statistically significant.

Kcnq1ot1 could be used as a prognostic indicator to determine left ventricular function (33-35).

Using microarray analysis, we previously found that lncRNA-FA2H-2 played an important role in the process of atherosclerosis, and lncRNA-FA2H-2 expression was significantly downregulated and closely associated with inflammatory responses in human atherosclerotic plaque tissues. Our previous study showed that the lesion areas were increased by lncRNA-FA2H-2 knockdown in the aortic root and aortic valve of $\mathrm{ApoE}^{-/-}$mice fed a high fat diet. Moreover, the intima of the aortic root and aortic valve were proliferative and disarranged, and had a large number of lipid-laden foam cells and cholesterol crystals in the lncRNA-FA2H-2 knockdown group. In addition, LncRNA-FA2H-2 knockdown increased VCAM-1, MCP-1, and IL-6 expressions in atherosclerotic lesions in $\mathrm{ApoE}^{-/-}$mice fed a high fat diet. Furthermore, LncRNAFA2H-2 attenuated the endothelial cell inflammation caused by OX-LDL. Based on the above findings, we propose that lncRNAFA2H-2 may be used as a potential inflammatory biomarker for CHD. In the current study, we verified the hypothesis by
TABLE 7 | Correlation analysis of LncRNA-FA2H-2 and clinical characteristics.

\begin{tabular}{lcc}
\hline & \multicolumn{2}{c}{ LncRNA-FA2H-2 } \\
\cline { 2 - 3 } & $\mathbf{R}$ & $\boldsymbol{P}$ \\
\hline Age & 0.07 & 0.44 \\
Gender & -0.80 & 0.06 \\
Hypertension & 0.04 & 0.38 \\
Diabetes & 0.209 & 0.073 \\
Smoking & 0.047 & 0.19 \\
Drinking & 0.05 & 0.381 \\
BMI & 0.43 & 0.21 \\
WBC & 0.128 & 0.089 \\
TG & 0.042 & 0.66 \\
TC & 0.218 & 0.053 \\
LDL-C & -0.11 & 0.238 \\
HDL-C & 0.05 & 0.547 \\
ApoA1 & -0.07 & 0.432 \\
\hline
\end{tabular}

TABLE 8 | Correlation analysis of LnCRNA-FA2H-2 and TNF- $\alpha$, MCP-1, VCAM-1, ICAM-1, IL-6.

\begin{tabular}{lcc}
\hline & \multicolumn{2}{c}{ LncRNA-FA2H-2 } \\
\cline { 2 - 3 } & $\mathbf{R}$ & $\boldsymbol{P}$ \\
\hline TNF- $\alpha$ & 0.22 & 0.82 \\
MCP-1 & -0.80 & 0.04 \\
VCAM-1 & -0.09 & 0.01 \\
ICAM-1 & -0.02 & 0.015 \\
IL-6 & -0.22 & 0.006 \\
\hline
\end{tabular}

examining the relative expressions of lncRNA-FA2H-2 and inflammatory markers in CHD patients, and the correlation between IncRNA-FA2H-2 and inflammatory responses. We found statistically significant expression levels of TG, HDL-C, ApoA1, and CRP in CHD patients, consistent with the results of previous studies (36). However, we also found that there was no statistically significant difference in diabetes, smoking, and drinking in CHD patients. We speculate that possible reasons for diabetes and smoking not being highly significant were as follows: (1) The enrolled patients were region-specific, only the patients in Henan Province were involved, and all of them were Han Chinese. (2) Due to regional specificities of the enrolled patients, there may have been deviations from large-scale studies that were not representative. Therefore, in the next study, we should increase the sample size and conduct a multi-center study. (3) Patient information was obtained from the hospital's 
electronic medical record system and lifestyles other than smoking and drinking were not recorded in the system.

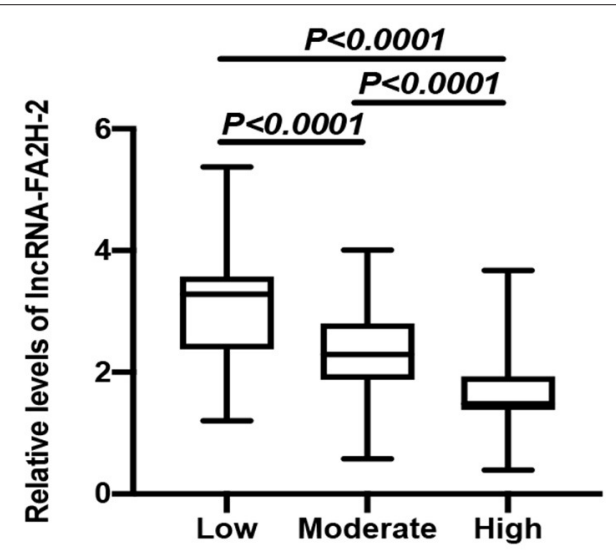

FIGURE 3 | Comparison of relative expressions of LnCRNA-FA2H-2 in the high risk, moderate risk, and low risk groups.

TABLE 9 | Correlation analysis of the Gensini score and LncRNA-FA2H-2.

\begin{tabular}{llc}
\hline & \multicolumn{3}{c}{ Gensini score } \\
\cline { 2 - 3 } & $\mathbf{R}$ & $\boldsymbol{P}$ \\
\hline LnCRNA-FA2H-2 & -0.642 & 0.032
\end{tabular}

TABLE 10 | Comparison of the sensitivities and specificities of TNF- $\alpha$, MCP-1, VCAM-1, ICAM-1, and IL-6.

\begin{tabular}{lccccc}
\hline & Sensitivity & Specificity & AUC & Yoden index & Cut-off \\
\hline TNF- $\alpha$ & $73 \%$ & $64 \%$ & $0.832(0.77,0.893)$ & 0.370 & 290.5 \\
MCP-1 & $59 \%$ & $79 \%$ & $0.731(0.653,0.809)$ & 0.380 & 396 \\
VCAM-1 & $72 \%$ & $83 \%$ & $0.822(0.757,0.887)$ & 0.550 & 264 \\
ICAM-1 & $75 \%$ & $65 \%$ & $0.794(0.715,0.874)$ & 0.400 & 201.5 \\
IL-6 & $55 \%$ & $90 \%$ & $0.760(0.685,0.834)$ & 0.450 & 328
\end{tabular}

To further investigate the role of inflammatory factors in CHD patients, we measured the expression levels of inflammationrelated indicators by ELISA and found that the levels of TNF- $\alpha$, MCP-1, VCAM-1, ICAM-1, and IL-6 were significantly elevated in CHD patients. In addition, the Gensini score represents the degree of coronary lumen stenosis. The results showed that the relative levels of TNF- $\alpha$, MCP-1, VCAM-1, ICAM-1, and IL-6 were associated with the Gensini score and degree of risk, suggesting that the expression levels of inflammatory markers correlated with the severity of CHD. The reason for these findings may be that the more severe the degree of coronary stenosis, the more intense the inflammatory response. Therefore, it can be tentatively concluded that the occurrence and development of CHD are inextricably linked to the inflammatory response. To further investigate the expression levels of lncRNAFA2H-2 in patients with CHD, we determined lncRNA-FA2H-2 expression using qRT-PCR and found that its expression levels were significantly decreased in CHD patients, which is consistent with our previous in vitro results. More importantly, lncRNAFA2H-2 negatively correlated with MCP-1, VCAM-1, ICAM-1, and IL-6, further confirming the potential of lncRNA-FA2H-2 as an inflammatory biomarker in CHD patients. Importantly, we found that lncRNA-FA2H-2 was correlated with the Gensini score, suggesting that lncRNA-FA2H-2 was related to the degree of stenosis and severity in CHD. In addition, the specificity of IncRNA-FA2H-2 was lower than that of other inflammatory indexes, which was considered to be due to the enrolled population of patients who underwent coronary angiography, and was different from a true healthy control group. However, the AUC was larger than the other indicators, indicating that the diagnosis had a certain advantage over the rest of the inflammatory indicators, but the specific mechanism was unclear. A possible reason for this is that lncRNA-FA2H-2 is more stable and $\operatorname{lncRNA}-\mathrm{FA} 2 \mathrm{H}-2$ may affect the occurrence and development of CHD through other pathways, although, further verification of this possibility is needed. In addition, using logistic analysis we found that lncRNA-FA2H-2 was a risk factor for $\mathrm{CHD}$, suggesting that lncRNA-FA2H-2 is expected to be a predictor for the development of CHD.

TABLE 11 | Comparison of the sensitivity and specificity of LncRNA-FA2H-2.

\begin{tabular}{|c|c|c|c|c|c|}
\hline & Sensitivity & Specificity & AUC & Yoden index & Cut-off \\
\hline LncRNA-FA2H-2 & $85 \%$ & $82 \%$ & $0.834(0.688,0.85)$ & 0.670 & 3.155 \\
\hline
\end{tabular}

TABLE 12 | Binary logistic regression analysis of CHD patients.

\begin{tabular}{|c|c|c|c|c|c|}
\hline & $\beta$ & Sx & Wald & RR (95\% Cl) & $P$ \\
\hline TNF- $\alpha$ & 0.08 & 0.02 & 11.549 & $1.0008(1.003,1.013)$ & 0.001 \\
\hline MCP-1 & 0.06 & 0.02 & 10.354 & $1.006(1.002,1.010)$ & 0.001 \\
\hline VCAM-1 & 0.10 & 0.03 & 11.624 & $1.010(1.004,1.015)$ & 0.001 \\
\hline ICAM-1 & 0.03 & 0.02 & 1.185 & $1.004(0.998,1.007)$ & 0.276 \\
\hline IL-6 & 0.09 & 0.02 & 11.797 & $1.009(1.004,1.014)$ & 0.001 \\
\hline LncRNA-FA2H-2 & -0.906 & 0.178 & 25.939 & $0.404(0.285,0.573)$ & 0.001 \\
\hline
\end{tabular}


The above results were limited by the following factors: (1) This was a single-center study with a small sample size, and the study subjects had differences in clinical baseline information. Therefore, a larger sample size is needed to verify the results. There are also more risk factors for $\mathrm{CHD}$, so our inclusion index was limited. (2) There may have also been operational errors during the study. (3) Although, lncRNAs exist in plasma and a variety of body fluids, the metabolic characteristics of specific lncRNAs need to be identified. In addition, the cellular origin of the detected circulating lncRNAs is difficult to determine, and we cannot yet eliminate the possibility that these IncRNAs are associated with other underlying diseases in patients with cardiovascular diseases. This could affect the specificity and sensitivity of lncRNA use in the diagnostic and prognostic determination of cardiovascular diseases. Although, studies have demonstrated that lncRNAs played an important role in various cardiovascular disease processes such as $\mathrm{CHD}$, heart failure, and hypertension, further, confirmation using larger sample sizes and plaque tissues from CHD patients is needed to determine whether lncRNA-FA2H2 can be an inflammatory biomarker and therapeutic target for CHD.

\section{REFERENCES}

1. Khamis RY, Ammari T, Mikhail GW. Gender differences in coronary heart disease. Heart. (2016) 102:1142-9. doi: 10.1136/heartjnl-2014-306463

2. Di Angelantonio E, Thompson A, Wensley F, Danesh J. Coronary heart disease. IARC Sci Publ. (2011) 363-86.

3. Ambrosio G, Tritto I. Interaction between the endothelium and blood cells in acute coronary syndromes. Ital Heart J. (2001) 2(Suppl. 3):43-4S.

4. Kinlay S, Selwyn AP, Libby P, Ganz P. Inflammation, the endothelium, and the acute coronary syndromes. J Cardiovasc Pharmacol. (1998) 32(Suppl. 3):S62-6.

5. Lheureux P, Even-Adin D, Askenasi R. Current status of antidotal therapies in acute human intoxications. Acta Clin Belg Suppl. (1990) 13:29-47. doi: 10.1080/17843286.1990.11718125

6. Hansson GK. Inflammation, atherosclerosis, and coronary artery disease. $N$ Engl J Med. (2005) 352:1685-95. doi: 10.1056/NEJMra043430

7. Inoue T, Uchida T, Yaguchi I, Sakai Y, Takayanagi K, Morooka S. Stent-induced expression and activation of the leukocyte integrin Mac-1 is associated with neointimal thickening and restenosis. Circulation. (2003) 107:1757-63. doi: 10.1161/01.CIR.0000060487.15126.56

8. Li H, Sun K, Zhao R, Hu J, Hao Z, Wang F, et al. Inflammatory biomarkers of coronary heart disease. Front Biosci. (2018) 10:185-96. doi: 10.2741/s508

9. Paraskevopoulou MD, Hatzigeorgiou AG. Analyzing MiRNALncRNA Interactions. Methods Mol Biol. (2016) 1402:271-86. doi: 10.1007/978-1-4939-3378-5_21

10. Wang J, Su Z, Lu S, Fu W, Liu Z, Jiang X, et al. LncRNA HOXA-AS2 and its molecular mechanisms in human cancer. Clin Chim Acta. (2018) 485:229-33. doi: 10.1016/j.cca.2018.07.004

11. Bhan A, Soleimani M, Mandal SS. Long non-coding RNA and cancer: a new paradigm. Cancer Res. (2017) 77:3965-81. doi: 10.1158/0008-5472.CAN-16-2634

12. Wu G, Cai J, Han Y, Chen J, Huang ZP, Chen C, et al. LincRNA-p21 regulates neointima formation, vascular smooth muscle cell proliferation, apoptosis, and atherosclerosis by enhancing p53 activity. Circulation. (2014) 130:145265. doi: 10.1161/CIRCULATIONAHA.114.011675

13. Shan K, Jiang Q, Wang XQ, Wang YN, Yang H, Yao MD, et al. Role of long non-coding RNA-RNCR3 in atherosclerosis-related vascular dysfunction. Cell Death Dis. (2016) 7:e2248. doi: 10.1038/cddis.2016.145

\section{DATA AVAILABILITY STATEMENT}

The original contributions presented in the study are included in the article/supplementary material, further inquiries can be directed to the corresponding author/s.

\section{ETHICS STATEMENT}

Written informed consent was obtained from the individual(s) for the publication of any potentially identifiable images or data included in this article.

\section{AUTHOR CONTRIBUTIONS}

FG and GL conceived and supervised the study and revised the manuscript. FG designed experiments and wrote the manuscript. FG and YS performed experiments. FG and BH analyzed the data. All authors read and approved the final manuscript.

\section{FUNDING}

This study was funded by the National Natural Sciences Foundation of China (Grant No. 82002199).
14. Yan Y, Zhang B, Liu N, Qi C, Xiao Y, Tian X, et al. Circulating long non-coding RNA UCA1 as a novel biomarker of acute myocardial infarction. Biomed Res Int. (2016) 2016:8079372. doi: 10.1155/2016/8079372

15. Holdt LM, Hoffmann S, Sass K, Langenberger D, Scholz M, Krohn K, et al. Alu elements in ANRIL non-coding RNA at chromosome 9p21 modulate atherogenic cell functions through trans-regulation of gene networks. PLoS Genet. (2013) 9:e1003588. doi: 10.1371/journal.pgen.1003588

16. Avazpour N, Hajjari M, Yazdankhah S, Sahni A, Foroughmand AM. Circulating HOTAIR RNA is potentially up-regulated in coronary artery disease. Genomics Inform. (2018) 16:e25. doi: 10.5808/GI.2018.16.4.e25

17. Aune TM, Crooke PS III, Patrick AE, Tossberg JT, Olsen NJ, Spurlock CF III. Expression of long non-coding RNAs in autoimmunity and linkage to enhancer function and autoimmune disease risk genetic variants. $J$ Autoimmun. (2017) 81:99-109. doi: 10.1016/j.jaut.2017.03.014

18. Beermann J, Piccoli MT, Viereck J, Thum T. Non-coding RNAs in development and disease: background, mechanisms, and therapeutic approaches. Physiol Rev. (2016) 96:1297-325. doi: 10.1152/physrev.00041.2015

19. Ounzain S, Micheletti R, Arnan C, Plaisance I, Cecchi D, Schroen B, et al. CARMEN, a human super enhancer-associated long non-coding RNA controlling cardiac specification, differentiation and homeostasis. J Mol Cell Cardiol. (2015) 89(Pt. A):98-112. doi: 10.1016/j.yjmcc.2015.09.016

20. Wang H, Gong H, Liu Y, Feng L. Relationship between lncRNA-Ang362 and prognosis of patients with coronary heart disease after percutaneous coronary intervention. Biosci Rep. (2020) 40:1-9. doi: 10.1042/BSR20201524

21. Chen L, Yang W, Guo Y, Chen W, Zheng P, Zeng J, et al. Exosomal lncRNA GAS5 regulates the apoptosis of macrophages and vascular endothelial cells in atherosclerosis. PLOS ONE. (2017) 12:e0185406. doi: 10.1371/journal.pone.0185406

22. Puthanveetil P, Chen S, Feng B, Gautam A, Chakrabarti S. Long non-coding RNA MALAT1 regulates hyperglycaemia induced inflammatory process in the endothelial cells. J Cell Mol Med. (2015) 19:1418-25. doi: 10.1111/jcmm.12576

23. Guo FX, Wu Q, Li P, Zheng L, Ye S, Dai XY, et al. The role of the LncRNA FA2H-2-MLKL pathway in atherosclerosis by regulation of autophagy flux and inflammation through mTOR-dependent signaling. Cell Death Differ. (2019) 26:1670-87. doi: 10.1038/s41418-018-0235-z

24. Rothenbacher D, Hoffmeister A, Brenner H, Koenig W. Physical activity, coronary heart disease, and inflammatory response. 
Arch Intern Med. (2003) 163:1200-5. doi: 10.1001/archinte.163. 10.1200

25. Tajfard M, Latiff LA, Rahimi HR, Moohebati M, Hasanzadeh M, Emrani AS, et al. Serum concentrations of MCP-1 and IL-6 in combination predict the presence of coronary artery disease and mortality in subjects undergoing coronary angiography. Mol Cell Biochem. (2017) 435:37-45. doi: 10.1007/s11010-017-3054-5

26. Reinhart K, Meisner M, Brunkhorst FM. Markers for sepsis diagnosis: what is useful? Crit Care Clin. (2006) 22:503-19, ix-x. doi: 10.1016/j.ccc.2006.03.003

27. Fritz JV, Heintz-Buschart A, Ghosal A, Wampach L, Etheridge A, Galas D, et al. Sources and functions of extracellular small RNAs in human circulation. Annu Rev Nutr. (2016) 36:301-36. doi: 10.1146/annurev-nutr-071715050711

28. Guo F, Tang C, Li Y, Liu Y, Lv P, Wang W, et al. The interplay of LncRNA ANRIL and miR-181b on the inflammation-relevant coronary artery disease through mediating NF-kappaB signalling pathway. J Cell Mol Med. (2018) 22:5062-75. doi: $10.1111 / \mathrm{jcmm} .13790$

29. Hu YW, Zhao JY, Li SF, Huang JL, Qiu YR, Ma X, et al. RP5-833A20.1/miR 382-5p/NFIA-dependent signal transduction pathway contributes to the regulation of cholesterol homeostasis and inflammatory reaction. Arterioscler Thromb Vasc Biol. (2015) 35:87-101. doi: 10.1161/ATVBAHA.114.304296

30. Kumarswamy R, Bauters C, Volkmann I, Maury F, Fetisch J, Holzmann A, et al. Circulating long non-coding RNA, LIPCAR, predicts survival in patients with heart failure. Circ Res. (2014) 114:1569-75. doi: 10.1161/CIRCRESAHA.114.303915

31. Piccoli MT, Gupta SK, Viereck J, Foinquinos A, Samolovac S, Kramer FL, et al. Inhibition of the cardiac fibroblast-enriched lncRNA Meg3 prevents cardiac fibrosis and diastolic dysfunction. Circ Res. (2017) 121:575-83. doi: 10.1161/CIRCRESAHA.117.310624
32. Yang $\mathrm{Y}$, Cai $\mathrm{Y}, \mathrm{Wu} \mathrm{G}$, Chen $\mathrm{X}$, Liu Y, Wang X, et al. Plasma long non-coding RNA, CoroMarker, a novel biomarker for diagnosis of coronary artery disease. Clin Sci. (2015) 129:675-85. doi: 10.1042/CS20150121

33. Qiu JJ, Lin XJ, Zheng TT, Tang XY, Zhang Y, Hua KQ. The exosomal long noncoding RNA aHIF is upregulated in serum from patients with endometriosis and promotes angiogenesis in endometriosis. Reprod Sci. (2019) 26:1590-602. doi: 10.1177/1933719119831775

34. Yang J, Huang $\mathrm{X}, \mathrm{Hu} \mathrm{F}, \mathrm{Fu} \mathrm{X}$, Jiang $\mathrm{Z}$, Chen K. LncRNA ANRIL knockdown relieves myocardial cell apoptosis in acute myocardial infarction by regulating IL 33/ST2. Cell Cycle. (2019) 18:3393-403. doi: 10.1080/15384101.2019.1678965

35. Sun F, Yuan W, Wu H, Chen G, Sun Y, Yuan L, et al. LncRNA KCNQ1OT1 attenuates sepsis-induced myocardial injury via regulating miR-192-5p/XIAP axis. Exp Biol Med. (2020) 245:620-30. doi: 10.1177/1535370220908041

36. Gentile M, Peluso R, Di Minno MND, Costa L, Caso F, de Simone B, et al. Association between small dense LDL and sub-clinical atherosclerosis in patients with psoriatic arthritis. Clin Rheumatol. (2016) 35:2023-9. doi: 10.1007/s10067-016-3344-4

Conflict of Interest: The authors declare that the research was conducted in the absence of any commercial or financial relationships that could be construed as a potential conflict of interest.

Copyright $\odot 2021$ Guo, Sha, Hu and Li. This is an open-access article distributed under the terms of the Creative Commons Attribution License (CC BY). The use, distribution or reproduction in other forums is permitted, provided the original author(s) and the copyright owner(s) are credited and that the original publication in this journal is cited, in accordance with accepted academic practice. No use, distribution or reproduction is permitted which does not comply with these terms. 